\title{
Utilização do Openredu como Ferramenta de Gestão do Conhecimento na Secretaria de Planejamento e Gestão de Pernambuco
}

\author{
Hugo Augusto Vasconcelos Medeiros1, Nathalia Luíza Farias da Silva ${ }^{1}$ \\ 1Instituto de Gestão Pública de Pernambuco \\ 50040-090, 1377 - Recife - PE - Brasil \\ hugo.vasconceloseseplag.pe.gov.br, nathalia.fariaseseplag.pe.gov.br
}

\begin{abstract}
Regarding a contribution to digital transformation in Public Administration, this paper presents the experience report centered on participant observation of Openredu implementing process as a tool for Knowledge Management in the Pernambuco State Department of Planning and Management. To that aim, describe the solution, define the concept and framework of Knowledge Management used, and present the trajectory of the implantation, detaching the preliminary outcomes and the observed patterns. Finally, there's an evaluation about the mature of the implantation, describing the positive impacts about over Knowledge Management main factors.
\end{abstract}

Resumo. Tendo em vista contribuir para a discussão sobre transformação digital na Administração Pública, este artigo apresenta um relato da experiência centrado nos observadores participantes da implantação da plataforma de ensino Openredu como ferramenta de Gestão do Conhecimento na Secretaria de Planejamento e Gestão de Pernambuco. Para isso, descreve a plataforma, define o conceito e a abordagem de Gestão do Conhecimento utilizados, e apresenta a trajetória de implantação, destacando os resultados preliminares e padrões observados. Por fim, há uma avaliação da maturidade da implantação, descrevendo os impactos positivos sobre os principais fatores da Gestão do Conhecimento.

\section{Introdução}

A Secretaria de Planejamento e Gestão de Pernambuco (Seplag) é uma unidade da administração direta responsável pelo planejamento, orçamento e por diferentes pautas da gestão estadual, como as diretrizes de gestão estratégica, de gestão por resultados e de desenvolvimento do modelo de gestão como um todo. A Seplag se organiza em secretarias executivas, dedicadas às áreas-fins de atuação: gestão estratégica e planejamento, gestão por resultados, apoio aos municípios, planejamento e gestão orçamentária, e coordenação geral.

Além disso, a Secretaria possui em sua estrutura o Instituto de Gestão Pública de Pernambuco Governador "Eduardo Campos" (IGPE), responsável por atividades transversais à Secretaria. O IGPE é organizado em dois núcleos: o Núcleo de Gestão do Conhecimento (NGC), responsável pelo Programa de Formação Continuada (PFC), pela 
pesquisa, difusão e gestão de conhecimento sobre gestão pública, gestão governamental, e inovação; e o Núcleo de Ciência de Dados (NCD), responsável pelo suporte à tomada de decisão, com base na gestão de dados e da informação, na avaliação e desenho de programas e políticas públicas prioritárias do Estado.

Nesta estrutura, e seguindo a diretriz de transformação digital (PERNAMBUCO, 2018), no segundo semestre de 2018, o IGPE iniciou a procura por um Ambiente Virtual de Aprendizagem (AVA) com o qual pudesse oferecer os cursos de seu PFC.

À época, a avaliação era de que 1) a oferta de ações presenciais de formação consumia bastante recursos operacionais, sobretudo tempo, com atividades burocráticas e não sistemáticas, tais como enviar, novamente, a cada curso, o matéria didático para todos os novos inscritos 2) não era possível garantir a capilaridade exigida em virtude da dispersão dos servidores do órgão pelas diversas pastas da gestão estadual, e algumas da gestão municipal; e 3) vários servidores repetiam os cursos de caráter mais técnico por não conseguirem aprender todos os conteúdos no ritmo em que as aulas eram ministradas.

Diante dessas dificuldades, optou-se pela transformação digital do serviço, tendo em vista padronizar a tecnologia de Gestão do Conhecimento, o que permitiria centralizar o mapeamento, o armazenamento e a comunicação dos conhecimentos, e oferecer ensino assíncrono em espaço, para os servidores distribuídos pelos órgãos, e em tempo, para que os servidores com dificuldades nos cursos técnicos pudessem aprender no seu próprio ritmo.

Normalmente, para casos de transformação digital como este, o padrão do Instituto de Gestão Pública de Pernambuco é buscar, dentro do governo e em repositórios públicos ou de código aberto (tais como alternativeto e joinup), ferramentas cujas características permitam torná-las soluções adaptáveis para resolver o problema em tela, e digitalizar o processo em questão.

Contudo, no caso do Openredu o processo foi um pouco diferente, pois alguns dos servidores envolvidos no projeto de transformação digital do PFC já haviam conhecido a ferramenta antes, quando trabalhavam na Secretaria de Educação de Pernambuco, onde a plataforma é utilizada para ensino híbrido nas escolas da rede estadual. Assim, a solução foi vista como adequada, por atender aos critérios não funcionais, uma vez que é escrita em código aberto já instanciado no Estado; e funcionais, por oferecer o ensino eletrônico e gestão da aprendizagem. Além disso, o benchmarking realizado à época, com opções semelhantes, como o Moodle, apontou a plataforma OpenRedu como mais adequada, sobretudo por suas características de rede social.

Então, este artigo descreve a experiência de implementação do Openredu na Seplag, abordando a perspectiva da transformação digital dos processos de negócio de formação continuada da Secretaria. A perspectiva metodológica é de um relato de experiência focado na recuperação da memória dos observadores participantes responsáveis pelo processo. Para organizar a escrita, na primeira parte, são discutidos os conceitos centrais da análise, tais como as características e funcionalidades do Openredu, a definição e abordagem de Gestão de Conhecimento usadas na implantação, e a perspectiva pedagógica relacionada à plataforma. Em seguida, faz-se um mergulho na trajetória da implementação da ferramenta, com foco nos desafios e limites experimentados na adoção, e apresentação de uma retrospectiva da implantação. $\mathrm{Na}$ 
conclusão, realiza-se uma análise da implementação a partir da proposta de Oliveira et al. (2011) de avaliação da maturidade de um modelo de Gestão do Conhecimento e mostra-se breve plano de continuidade da implementação, focado na sensibilização dos servidores quanto às vantagens da ferramenta, oferecendo, inclusive, orientações para adoção da plataforma em outros órgãos.

\section{Transformação Digital, Openredu e Gestão do Conhecimento.}

Tanto na estratégia de governança digital federal (BRASIL, 2018), quanto na estadual (PERNAMBUCO, 2018), a perspectiva de transformação digital aparece com força, indicando a necessidade de um alinhamento estratégico para a construção de um Governo Digital que, usando de TIC, desenvolva a própria administração pública e ofereça melhores serviços para a população.

Neste contexto, é fundamental o papel da Seplag, enquanto Secretaria responsável pelo planejamento, pela gestão e pelo orçamento para as iniciativas públicas, pois, como afirmam Kane et al. (2015), a transformação digital é mais uma questão de estratégia do que de tecnologia, uma vez que cabe à estratégia criar um alinhamento e uma mudança organizacional que permitam a transformação, a partir das tecnologias que, em muitos casos, já se encontram disponíveis.

Então, a principal contribuição da Seplag para a transformação digital tem sido o mapeamento das iniciativas públicas e o posterior planejamento e suporte à modificação dos serviços analógicos em serviços digitais. Desse modo, a busca pelo Openredu alinha-se à diretriz de transformação digital e a um esforço da Seplag induzir a modificação de seus próprios serviços internos.

Outro ponto importante para compreender a adoça da plataforma é a perspectiva e a maturidade de Gestão de Conhecimento $(\mathrm{GC})$ presentes na Seplag à época. De acordo com Oliveira et al. (2011), a implementação de um modelo de Gestão do Conhecimento envolve quatro fatores:

- Contexto interno: cultura organizacional, suporte da alta administração, estrutura organizacional;

- Conteúdo: benefícios, objetivos, alinhamento estratégico, mapeamento de conhecimento crítico, tácito e explícito;

- Processo: comunicação, tecnologia, treinamento, tempo, recompensa, recrutamento, orçamento, disseminação, armazenamento, criação; e

- Contexto externo: parceiros, legislação, competidores, fornecedores, clientes.

Assim, para desenvolver a GC é necessário criar um contexto interno favorável, desenvolver o conteúdo que será gerenciado, os processos através dos quais se efetivará a gestão, e, não menos importante, considerar os aspectos externos que podem influenciar a adoção - o que, no caso público, é muito importante, em virtude do sentido estrito de legalidade ao qual ela se submete.

Além disso, é preciso escolher uma abordagem de GC. No momento da adoção da plataforma, foi escolhida a abordagem de "aprendizagem e educação" (FUKUNAGA, 2018), que pode ser definida como um tipo de Gestão do Conhecimento em que as ações de encontrar, organizar e compartilhar conhecimento acontecem com 
foco no fornecimento de ações de capacitação, de formação continuada (JANNUZZI, 2016).

A avaliação dos fatores indicava que havia problemas com o mapeamento do conhecimento, que muitas vezes precisava ser repetido; com a sua comunicação, já que toda ela ocorria por troca de e-mails e por telefone; tecnologia, pois eram usadas diversas tecnologias diferentes para gerenciamento; treinamento e tempo, porque muitas vezes as formações tinham de ser repetidas ou recursadas, sobretudo as formações do eixo técnico; disseminação, haja vista a dificuldade de levar treinamentos para outros setores, distantes da Seplag; e no armazenamento, uma vez que os objetos educacionais eram guardados na rede compartilhada da secretaria.

Nessa perspectiva, o Openredu poderia ser a solução, pois permitiria agir sobre os pontos onde a GC da Seplag apresentava baixa maturidade. Se não, vejamos: de acordo com Gomes et al. (2017), o Openredu é um software livre (GPL2), que oferece, ao mesmo tempo, um Ambiente Virtual de Aprendizagem (AVA), um Sistema de Gestão da Aprendizagem (SGA) e uma rede social; o que propicia, no mesmo ambiente, as funções de ensino, de gestão do que se ensina e aprende, de compartilhamento de informações e conhecimentos, e de engajamento dos estudantes e professores (FERREIRA, 2017).

Então, a própria adoção da ferramenta já daria maturidade ao elemento tecnologia, padronizando-a. Ademais, as funcionalidades presentes no AVA permitiriam amadurecer em relação a treinamento, tempo, disseminação $\mathrm{e}$ armazenamento; as de SGA também fortaleceriam as de treinamento; e as de rede social, as de comunicação e disseminação.

Também se deve destacar que o Openredu atendia plenamente aos principais requisitos não funcionais elencados para a transformação das ações de capacitação presenciais em digitais: 1) adoção de um software livre, em virtude de questões como custo, licenciamento e liberdade de customização e utilização (OSÓRIO ET AL., 2005); 2) preferência por uma plataforma de fácil apreensão e uso, uma vez que os professores e estudantes do PFC são servidores públicos e realizam as formações em seu horário de trabalho, havendo pouco tempo disponível para alimentar a plataforma, para navegar entre diferentes plataformas ou para aprender como usar uma ferramenta complexa.

\section{Trajetória da Implantação do Openredu: desafios, limites e usos}

A implantação do Openredu começou com a abertura de chamado para preparação da instância junto à Agência Estadual de Tecnologia da Informação (ATI), em 18/05/2018; com conclusão do processo um mês depois, em 19/06/2018.

Neste momento, a instância parecia estável e os testes de uso da ferramenta foram iniciados. Contudo, em 20/08/2018, foram identificados problemas relativos a funcionalidades básicas da ferramenta, como o envio de e-mails de amizade, convite para a ferramenta, recuperação de senhas, etc.. o upload de arquivos; e a busca por ambientes.

A principal dificuldade na resolução dessas questões foi o fato de por a ATI não ser uma fábrica de software, não realizar modificações nem customizações nas ferramentas. A solução encontrada foi solicitar apoio na comunidade da ferramenta, e 
passar a orientação de modificação para a ATI apenas implementar. Com esse suporte da comunidade, as correções foram finalizadas em 03/10/2018.

Em paralelo aos testes, a plataforma foi sendo utilizada pela equipe responsável pelo PFC, tendo em vista observar se ela atendia às regras de negócio necessárias à oferta de curso. Neste momento, retomando o modelo de Oliveira et al (2011), observamos que o elemento legislação, do fator contexto externo, foi observado que não havia compatibilidade entre as regras de negócio expressas na legislação vigente no Estado sobre instrutoria e as funcionalidades disponíveis na Plataforma, fazendo com que sua adoção diminuísse a maturidade neste elemento, e no elemento tempo, já que seria necessário realizar atividades por fora da plataforma para atender aos requisitos legais.

Em suma, a ferramenta não poderia ser usada diretamente para Educação à Distância $(\mathrm{EaD})$, em virtude de ela não oferecer funções de gestão administrativa dos cursos (como, por exemplo, certificação), de oferecer pouca possibilidade de customização de leiaute e de revisão, por exemplo, das ordens e dos materiais carregados para o servidor.

Neste ponto, a avaliação foi mista, pois 1) da perspectiva da recepção da solução, a preparação da instância foi relativamente simples, durando no total cerca de 70 dias (incluindo o período de testes e de correções), e o fato de a comunidade da plataforma ser ativa e solícita mostrou-se uma vantagem adicional; 2) do ponto de vista da solução do problema, a ferramenta não servia ao objetivo inicial, que era transformar digitalmente o serviço de oferta de cursos, uma vez que ela diminuiria a maturidade nos elementos tempo e legislação.

Assim, a secretaria tinha à disposição uma solução interessante, com zero custo contábil, mas que não resolvia o problema. Isso fez com que a ferramenta permanecesse inativa durante o final do ano de 2018.

Em 2019, contudo, houve uma troca na gestão do Instituto e na equipe responsável pela formação continuada, que trouxe consigo algumas mudanças de perspectiva:

- Expandir o uso apenas da abordagem de gestão de conhecimento por "aprendizagem e educação", para incluir as abordagens de GC por "interação e colaboração", focada na localização, compartilhamento e consulta de conhecimento, e por "informação e conteúdo", preocupada com o registro, acesso e uso das informações e conteúdos disponíveis (FUKUNAGA, 2018);

- Executar as formações a partir de trilhas de aprendizagem, as quais podem ser definidas como "Conjuntos integrados e sistemáticos de ações de desenvolvimento, que recorrem a múltiplas formas de aprendizagem" (MURASHIMA, 2011), formas essas organizadas em repositórios.

Assim, com as mudanças de perspectiva, o problema deslocou-se de como reduzir os custos operacionais (treinamento, tempo e comunicação), aumentar a capilaridade dos treinamentos (disseminação) e oferecer ensino assíncrono (treinamento), para como localizar e compartilhar conhecimento (mapeamento de conhecimento), através do registro e uso dos objetos de aprendizagem das trilhas (armazenamento). 
Essas mudanças fizeram com que o Openredu voltasse para o jogo, uma vez que a abordagem por interação e colaboração não possui legislação, permitindo que ela seja feita de acordo com as regras de negócio decididas pela própria equipe. Então, a adoção continuaria trazendo os mesmos resultados esperados, sem o impacto negativo sobre os elementos tempo e legislação.

Ao oferecer funções de gerenciamento de aprendizagem, tais como organizar todos os objetos de aprendizagem no mesmo lugar (GOMES ET AL., 2017); e por aceitar diversos tipos de objetos de aprendizagem, o Openredu tornou-se uma opção perfeita de repositório educacional, atendendo a abordagem de GC por informação e conteúdo.

Além disso, sua estrutura de rede social, que tende a aumentar o engajamento dos estudantes, e as interações entre eles e entre eles e os professores, mostrou-se uma ótima possibilidade de fazer as pessoas entrarem em contato e compartilharem seus conhecimentos (FERREIRA, 2017).

Por isso, a ferramenta transbordou seu sentido original, e passou a ser usada não somente para o PFC, mas para outras ações de capacitação da secretaria, e do próprio Governo, de forma mais ampla.

Retomada a decisão de utilização da plataforma, foram retomados os teste. Inicialmente, a própria equipe do Instituto de Gestão usou a ferramenta para compartilhar os conteúdos e realizar ensino híbrido em dois cursos de formação interna da equipe: Modelagem de Dados e Arquitetura Corporativa. Os resultados foram bons, atingindo a esperada redução nas trocas de mensagens e materiais, e facilitando a gestão da aprendizagem, uma vez que os estudantes têm se inscrito sozinhos nos cursos, através da sua rede de contatos e baixado os materiais de forma autônoma.

Além do uso interno, a equipe do PFC já usou a ferramenta com três cursos abertos ao público do PFC e uma oficina: Lei de Responsabilidade Fiscal, Design Thinking e Avaliação de Políticas com Inteligência Artificial; e a oficina Sistema Eletrônico de Informações. Nesses casos, os resultados foram semelhantes, uma vez que a equipe do PFC e os professores reduziram o envio de informações e materiais por email para somente 1 e-mail - o de confirmação da matrícula e indicação do site do OpenRedu onde a disciplina está hospedada. Nesses quatro casos, em virtude do material compartilhado, a matrícula nas disciplinas é moderada pela equipe do PFC.

Houve ainda um uso externo, numa perspectiva de Massive Open Online Course (MOOC), em que o ambiente chamado Espaço de Inovação foi aberto para todos os participantes de um evento de inovação governamental. Embora a perspectiva fosse de uso massivo, uma vez que o ambiente foi aberto para inscrição sem moderação, a porcentagem de membros no ambiente em relação ao total de inscritos no evento presencial foi relativamente baixa (o que ainda não podemos generalizar, em virtude de ter sido realizado este uso em apenas um evento), como se vê no quadro-resumo abaixo, com os indicadores dos três ambientes de ensino usados na plataforma:

\begin{tabular}{|l|l|c|c|c|c|}
\hline Ambiente & Disciplinas & $\begin{array}{c}\text { Ação } \\
\text { Presencial? }\end{array}$ & Aulas & Membros & $\begin{array}{c}\% \\
\text { membros/inscritos } \\
\text { na ação presencial }\end{array}$ \\
\hline
\end{tabular}




\begin{tabular}{|c|c|c|c|c|c|}
\hline PFC & Programação & Não houve & 4 & 24 & - \\
\hline PFC & $\begin{array}{l}\text { Análise de } \\
\text { Políticas } \\
\text { Públicas } \\
\end{array}$ & Curso & 6 & 24 & $120 \%$ \\
\hline PFC & $\begin{array}{ll}\text { Análise } & \text { de } \\
\text { Negócios } & \end{array}$ & Curso & 11 & 12 & $171 \%$ \\
\hline PFC & Inovação & Curso & 2 & 12 & $60 \%$ \\
\hline PFC & $\begin{array}{l}\text { Gestão do } \\
\text { Conhecimento }\end{array}$ & Não houve & 4 & 7 & - \\
\hline $\mathrm{PFC}$ & $\begin{array}{l}\text { Modelagem de } \\
\text { Dados }\end{array}$ & Curso & 5 & 7 & $100 \%$ \\
\hline PFC & $\begin{array}{l}\text { Legislação } \\
\text { Orçamentária }\end{array}$ & Curso & 1 & 6 & $30 \%$ \\
\hline $\begin{array}{l}\text { Espaço de } \\
\text { Inovação }\end{array}$ & Inovação & Evento & 14 & 10 & $30 \%$ \\
\hline $\begin{array}{l}\text { Espaço de } \\
\text { Inovação }\end{array}$ & Design Thinking & Evento & 4 & 10 & $30 \%$ \\
\hline $\begin{array}{l}\text { Espaço de } \\
\text { Inovação }\end{array}$ & Métodos Ágeis & Evento & 6 & 10 & $30 \%$ \\
\hline $\begin{array}{l}\text { Espaço de } \\
\text { Inovação }\end{array}$ & $\begin{array}{l}\text { Economia } \\
\text { Comportamental }\end{array}$ & Evento & 3 & 10 & $30 \%$ \\
\hline SEI & Oficina SEI & Oficina & 3 & 5 & $3 \%$ \\
\hline
\end{tabular}

Como se observa, a ferramenta está povoada com 12 disciplinas, as quais têm número de aulas oscilando entre 1 e 14 , e um total de alunos entre 5 e 24 . A análise qualitativa dos perfis das disciplinas mostrou que o total de aulas não é um indicativo fidedigno do total de conteúdo, uma vez que as disciplinas com poucas aulas estão com os conteúdos concentrados, com todas as apresentações sendo oferecidas em aula única. Quanto ao total de participantes, observamos que quando as disciplinas são ferramentas de ensino eletrônico de uma aula que também ocorre presencialmente, o número de inscritos tende a ser maior; enquanto a oficina apresentou porcentagem muito baixa de inscritos (contudo, não temos números ainda para avaliar se essa é uma tendência das oficinas).

Finalmente, foi observado ainda que a ferramenta serve ao propósito de repositório de objetos de conhecimento explícito, ainda que não conectados a ações de formação e capacitação. Assim, o armazenamento e a disseminação de objetos de conhecimento de reuniões de construção de produtos também estão realizados através da ferramenta, já que ele permite controlar o acesso aos objetos e também, para este caso, reduz o envio de e-mails.

Assim, chegamos a julho com 4 ambientes ativos, 12 disciplinas, 52 membros, e três macro usos distintos: oferta de objetos educacionais de cursos presenciais, como 
ensino híbrido, com matrícula controlada; ambiente de inscrição aberta e autoinstrução; compartilhamento de materiais usados em reuniões, de forma controlada, sem atividades educacionais atreladas.

\section{Avaliação e Plano de Ação}

Nesta seção, é apresentada a avaliação do desenvolvimento da plataforma tomando por bases os fatores elencados por Oliveira et al (2011), quais sejam contexto interno, conteúdo, processo e contexto externo.

Relembrando o que já foi destacado anteriormente, deve-se considerar que as organizações caminham através de cinco níveis, de 0 a 4, de acordo com a maturidade do fator. Em resumo, podemos dizer:

- Nível 0 - o fator não existe (por exemplo, não há tecnologia), é irregular ou superficial (por exemplo, um suporte administrativo errático), ou, de acordo com o fator analisado, são mal vistas / mal avaliadas (uma legislação ou que dificulta a Gestão do Conhecimento).

- Nível 1 - o fator ocorre com regularidade mas é incipiente, ou acontece com considerável esforço / desperdício, dependendo de um agente específico, por exemplo.

- Nível 2 - o fator funciona com o mínimo de padronização e regularidade devidas;

- Nível 3 - o fator passa a ocorrer de maneira fluída, desenvolvendo-se na organização, trasbordando para outros setores;

- Nível 4 - o fator acontece de forma plena, alcançado a organização, seu ambiente interno e externo.

Evidentemente, o Openredu não pode responder a todos os fatores mapeados, mas com a sua utilização foram percebidos alguns avanços, detalhados a seguir:

- Mapeamento de Conhecimento N2: repositório de conhecimentos explícitos e permitir a partilha de conhecimento tácito através dos seus fóruns, permitindo, inclusive, que cada estudante trilhe seu próprio caminho de aprendizagem (MURASHIMA, 2011).

- Comunicação N2: partilha padronizada e centralizada, com baixo retrabalho, e potencial para atingir o nível 3 , caso os inscritos passem a utilizar na sua rotina a comunicação através da ferramenta para aprenderem e tirarem dúvidas sobre rotinas, etc.

- Tecnologia N2: ferramenta padronizada e disponível em qualquer tempo.

- Treinamento N1: pois ainda há um número pequeno de objetos na plataforma, contudo, conforme o número torne-se maior, espera-se atingir o N2.

- Tempo N2: já que o tempo gasto com partilha dos materiais diminuiu e se padronizou.

- Armazenamento N2: armazenamento padronizado com repositório centralizado; 
- Disseminação N2: pois a ferramenta tem permitido alcançar mais facilmente agentes de outras secretarias, etc. Inclusive, já foram observadas 2 inscrições de pessoas que não estavam na divulgação do ambiente ou disciplina.

Além desses resultados, observa-se potencial para atingir o nível 2 no fator benefícios, em virtude da possibilidade de gamificação da recompensa e de utilização de formas mais ativas de ensino, como o híbrido e a sala de aula invertida.

Contudo, há que se lidar com as dificuldades e apresentar resultados, para que a ferramenta não seja mal vista ou mal avaliada. As principais dificuldades têm sido de sensibilização e engajamento para o uso da ferramenta, e as reclamações, referentes à experiência do usuário na ferramenta, sobretudo com as telas monocromáticas, e links pequenos para ,por exemplo, baixar, os materiais de apoio dentro dos cursos.

A criação da conta, inscrição nos cursos, uso dos objetos, etc. tem sido fáceis, não necessitando, na maioria dos casos, de treinamento específico. Porém, tem-se mostrado necessária a sensibilização em relação ao ensino eletrônico e à possibilidade de utilização da ferramenta para criar comunidades de aprendizagem, colocando o aluno como protagonista do processo de ensino-aprendizagem, já que os pedidos de envio de material por e-mail ainda são frequentes.

Deve-se considerar, neste ponto, que, conforme citado na introdução, a transformação digital é muito mais um processo de estratégia do que de tecnologia em si (KANE ET AL., 2015). Então, para continuar avançando com a GC numa perspectiva digital, é necessário cuidar, sobretudo, do fator contexto interno, sensibilizando os servidores e a alta administração, e criando formas de integrar os diferentes setores.

Assim, planeja-se para o fim de 2019 um grande evento específico de lançamento, sensibilização e engajamento para a ferramenta, no qual serão explicados os benefícios de usar trilhas de aprendizagem, e os benefícios de usar o Openredu. Além disso, pretende-se manter a estratégia de que os novos cursos para o público geral continuem usando a ferramenta, pelo menos, para armazenar os objetos educacionais usados nas ações presenciais - sendo o ideal a utilização para ensino híbrido; bem como a criação de MOOCS, e a disponibilização de materiais de reuniões. Com isso, esperase, avançar para que todos os fatores relacionados à ferramenta estejam pelo menos no nível 2, construindo o nível 3 naqueles fatores que a ferramenta permitir avanço adicional, como na comunicação.

Fazendo uma avaliação retrospectiva e prescritiva, para outros órgãos que possivelmente desejem adotar transformação digital em sua Gestão do Conhecimento, devemos dizer que a opção por uma ferramenta integrada (AVA + SGA + Rede Social) de código livre foi acertada em virtude de possibilitar uma tecnologia padronizada e centralizada, obtida apenas a custo de oportunidade.

Além disso, algumas boas lições aprendidas foram: 1) pensar não somente do problema para a solução, mas da solução para o problema, já que o Openredu não atendia ao esperado, mas possuía muitas outras vantagens, que puderam ser colhidas por meio da alteração da abordagem de Gestão do Conhecimento; 2) buscar uma solução livre cuja comunidade seja ativa e solidária, pois isso reduziu bastante o tempo de instalação, customização e manutenção, além de permitir usar a ferramenta de forma mais significativa, e já ter, inclusive, um banco de talentos para treinamentos e contratações relativas à ferramenta; 3) o fator legislação deve ser sempre levado 
fortemente em consideração, já que a sua baixa maturidade pode diminuir a maturidade de outros fatores, ou, até mesmo, inviabilizar a solução; 4) os fatores de contexto interno não inviabilizam a solução, mas seguram a maturidade de outros fatores, pois tem poder de criar um lock-in, travando a Gestão do Conhecimento a um ou a poucos setores.

\section{Referências}

Brasil. (2018) Estratégia de Governança Digital - EGD - Transformação Digital: Cidadania e Governo 2016-2019 - Versão Revisada. Brasília, Brasil, Ministério do Planejamento, Desenvolvimento e Gestão.

Ferreira, J. (2017) Análise da Efetividade da Comunicação da Rede Social Educativa Openredu em Comunidades de Prática. Recife, Brasil, Dissertação de Mestrado, Programa de Pós-graduação em Ciência da Computação, Centro de Informática, Universidade Federal de Pernambuco.

Fukunaga, F. (2019) “Abordagens em GC", In: Blog da SBGC, http://www.sbgc.org.br/blog/abordagens-em-gc, fevereiro.

Gomes, S. et al. (2017) "Ensino e Aprendizagem com a rede social Openredu": Tecnologias em EaD: Métodos e Práticas, Leite, J., Sousa, H. e Oliveira, E., João Pessoa-PB, Brasil, Editora da UFPB, p. 9-35.

Jannuzzi, C. et al. (2016) "Gestão do conhecimento: um estudo de modelos e sua relação com a inovação nas organizações", In: Perspectivas em Ciência da Informação, 21(1), 97-118, janeiro/março.

Kane, G. et al. (2015) "STRATEGY, NOT TECHNOLOGY, DRIVES DIGITAL TRANSFORMATION”, In: MIT SMR, https://sloanreview.mit.edu, julho.

Murashima, M. (2011) "Universidades Corporativas: as trilhas em meio a novos caminhos", In: Revista FGV Online, 1(2), 4-23, http://bibliotecadigital.fgv.br/ojs/index.php/revfgvonline/article/view/19871, abril.

Oliveira, M. et al. (2011) "Proposta de um modelo de maturidade para Gestão do Conhecimento", In: KM‥ Revista de Gestão dos Países de Lingua Portuguesa, 10(4), 14-25. http://www.scielo.mec.pt/scielo.php?script=sci_arttext\&pid=S1645$44642011000300003 \& \operatorname{lng}=\mathrm{pt \&}$ tlng=pt.

Osório, T. (2005) "UTILIZAÇÃO DE SOFTWARE LIVRE EM ORGÃOS PÚBLICOS", In: Anais do II Simpósio de Excelência em Gestão e Tecnologia SEGeT'2005, https://www.aedb.br/seget/arquivos/artigos05/360_Artigo_SL_Completo.pdf.

Pernambuco. (2018) LEI No 16.379, DE 6 DE JUNHO DE 2018 - Altera a Lei $n^{\circ}$ 12.985, de 2 de janeiro de 2006, que dispõe sobre o Sistema Estadual de Informática de Governo - SEIG. Recife, Brasil, Assembleia Legislativa de Pernambuco. 